\title{
28 Research Soure \\ Repeated Cardiac Arrest Following Emergency Carotid Artery Stenting.- A Case Report.
}

\section{Qinqin Wang ( $\sim 354521416 @ q q . c o m)$}

Zhejiang Hospital of Traditional Chinese Medicine https://orcid.org/0000-0003-1785-6609

\section{Huihui Wu}

Taian Hospital of Traditional Chinese Medicine

\section{Bin Hou}

Taian Hospital of Traditional Chinese Medicine

\section{Case report}

Keywords: cardiac arrest, carotid artery, stenting, carotid sinus reflect

Posted Date: October 18th, 2021

DOI: https://doi.org/10.21203/rs.3.rs-942105/v1

License: (1) This work is licensed under a Creative Commons Attribution 4.0 International License. Read Full License 


\section{Abstract}

Background: Due to carotid sinus response, blood pressure reduction and bradycardia during and after carotid stent implantation was reported frequently, in contrast, repeated cardiac arrest is rare but fatal unless the heart can be started quickly.

Case introduction: A 61-year-old male presented with severe left hemiplegia, aphasia, central facial palsy and gaze. Emergency surgery under general anesthesia was finally scheduled in view of possible severe cerebral arterial stenosis. The patient underwent ipsilateral ICA stenting after balloon dilatation and was transferred to intensive care unit(ICU). His heart rate tended to decrease sharply and then cardiac arrest was noted on electrocardiograph(ECG) monitor. Emergency cardiopulmonary resuscitation(CPR) was started and repeated three times due to persistent symptoms. Coronary angiography was postponed for 15 days until neurological recovery. Multivessel disease with severe stenosis one of them was confirmed and percutaneous coronary intervention ( $\mathrm{PCl}$ ) was performed. The patient was discharged on the 20th day of admission with only slight neurological deficits.

Conclusion: We tend to attribute the causes of repeated cardiac arrest in this case to carotid sinus response induced by stenting and serious coronary stenosis.

\section{Background}

Carotid artery stenting is an effective therapy to achieve recanalization particularly in fatal or disabling stroke with symptomatic carotid stenosis. Compared with the reduction in blood pressure and heart rate following stenting, repeated cardiac arrest which can lead to serious sequelae or even death has been rarely reported.

OBJCETIVE: To present a case of repeated cardiac arrest after emergency carotid artery stenting and try to explore the possible causes.

\section{Case Description}

A 61-year-old male presented with severe left hemiplegia, aphasia, central facial palsy and gaze. The National Institute of Health Stroke scale was 12. Head computed tomography(CT) scan without contrast injection revealed acute ischemia in the right lobe. Electrocardiogram showed a ventricular rate of 70 beats per minute and inferior ST-segment depression. The patient was subsequently treated with full-dose urokinase intravenous thrombolysis, but it was ineffective. Emergency surgery under general anesthesia was finally scheduled in view of possible severe cerebral arterial stenosis. Digital subtraction angiography showed right internal carotid artery(ICA) origin stenosis of $>95$ percent and right middle carotid artery (MCA) total occlusion from the origin. Complete recanalization of right MCA was obtained after one pass of a stent retriever, despite embolism of distal branches. The patient underwent ipsilateral ICA stenting after balloon dilatation and was transferred to intensive care unit(ICU). Postoperative cardiovascular deterioration was observed immediately after ICU admission. His heart rate tended to 
decrease sharply and then cardiac arrest was noted on electrocardiograph(ECG) monitor. Emergency cardiopulmonary resuscitation(CPR) was started and repeated three times due to persistent symptoms. Temporary ST-segment elevation was noted on ECG which followed. Coronary angiography was postponed for 15 days until neurological recovery. Multivessel disease with severe stenosis one of them was confirmed and percutaneous coronary intervention $(\mathrm{PCl})$ was performed. The patient was discharged on the 20th day of admission with only slight neurological deficits.

\section{Conclusion}

Repeated cardiac arrest is rare but fatal unless the heart can be started quickly. We tend to attribute the causes of repeated cardiac arrest in this case to carotid sinus response induced by stenting and serious coronary stenosis.

\section{Abbreviations}

CT: Computed tomography; ICA: Internal carotid artery; MCA: Middle carotid artery; ICU: Intensive care unit; ECG: Electrocardiograph; CPR: Emergency cardiopulmonary resuscitation; PCl: Percutaneous coronary intervention.

\section{Declarations}

\section{Ethics approval and consent to participate}

The ethics committee of Taian Hospital of Traditional Chinese Medicine approved the study.

\section{Consent for publication}

All of the authors agree to the publication of the article.

\section{Availability of data and materials}

All data generated or analysed during this study are included in this published article and its supplementary information file.

\section{Competing interests}

The authors declare that they have no competing interest.

\section{Acknowledgments}

The authors gratefully acknowledge the physicians involved in the treatment.

\section{Authors' contributions}


Qinqin Wang analyzed and interpreted the patient data and was a major contributor in writing the manuscript. Huihui Wu and Bin Hou performed the histological examination of the patient. All authors read and approved the final manuscript.

\section{Supplementary Files}

This is a list of supplementary files associated with this preprint. Click to download.

- CAREchecklistEnglish2013.pdf

- supplementarymaterial.doc 